\title{
TUMOR IMPLANTATION AFTER COMPUTED TOMOGRAPHY-GUIDED BIOPSY OF LUNG CANCER
}

\author{
Yannis Raftopoulos, MD, Warren W. Furey, MD, Daniel J. Kacey, MD, and Francis J. Podbielski, MD, Chicago, Ill
}

Approximately 150,000 solitary pulmonary nodules are discovered annually in adults older than age 50 years, with $40 \%$ to $50 \%$ being malignant. ${ }^{1}$ Histologic diagnosis is often impossible by radiographic assessment or bronchoscopy alone. The evolution of minimally invasive techniques like transthoracic biopsy guided by computed tomography (CT) and videoassisted thoracoscopic surgery (VATS) has offered alternatives other than open surgical biopsy for preoperative diagnosis.

Although CT-guided biopsy and VATS are effective and valuable thoracic diagnostic procedures, preservation of oncologic principles remains an issue. Despite its rarity, tumor dissemination after transthoracic biopsy or VATS ${ }^{2}$ is a potential risk. The significance of tumor seeding along the needle or port tract with respect to management of lung lesions suspected to be malignant, as well as the extent of treatment required and the overall impact on the patient's prognosis, has yet to be defined.

Clinical summary. A 51-year-old man had Mycobacterium tuberculous peritonitis. A chest radiograph showed a $1.5 \mathrm{~cm}$ lung nodule in the left upper lobe. Medical history was noteworthy for pancreatitis, hypertension, and a 50 pack-year smoking history. Physical examination showed no abnormalities. CT scan of the chest and abdomen confirmed the presence of the mass and showed no mediastinal lymphadenopathy and no hepatic or adrenal masses. Bronchoscopic examination showed no intraluminal masses and washings were inconclusive. A CT scan-guided percutaneous biopsy was performed with a 0.8 -mm diameter (20 gauge) screw tip needle (Rotex catheter, Ursus Corporation, Stockholm, Sweden), with two passes being made into the suspicious area. Pathologic study yielded malignant epithelial cells consistent with non-small cell cancer.

The patient underwent a left upper lobectomy and mediastinal lymphadenectomy. A pathologic diagnosis of moderately differentiated adenocarcinoma (tumor size $1.2 \times 1.1 \times$ $1.0 \mathrm{~cm}$ ) with normal (negative) lymph nodes was returned and classified as T1 N0 MX lung cancer. The patient did well after the operation and received no adjuvant therapy.

From the Department of Thoracic and Cardiovascular Surgery, Mercy Hospital and Medical Center, Metropolitan Group Hospitals General, Surgery Program at Chicago, Chicago, Ill.

Received for publication Dec 13, 1999; accepted for publication Jan 24, 2000.

Address for reprints: Francis J. Podbielski, MD, C.D.N. Surgical Associates, 2515 N Clark St, Suite 903, Chicago, IL 60614-2720 (E-mail: Fjpmeteu@aol.com).

J Thorac Cardiovasc Surg 2000;119:1288-9

Copyright $\odot 2000$ by The American Association for Thoracic Surgery $0022-5223 / 2000 \$ 12.00+0 \quad \mathbf{1 2 / 5 4} / \mathbf{1 0 5 8 8 8}$

doi: $10.1067 / \mathrm{mtc} .2000 .105888$

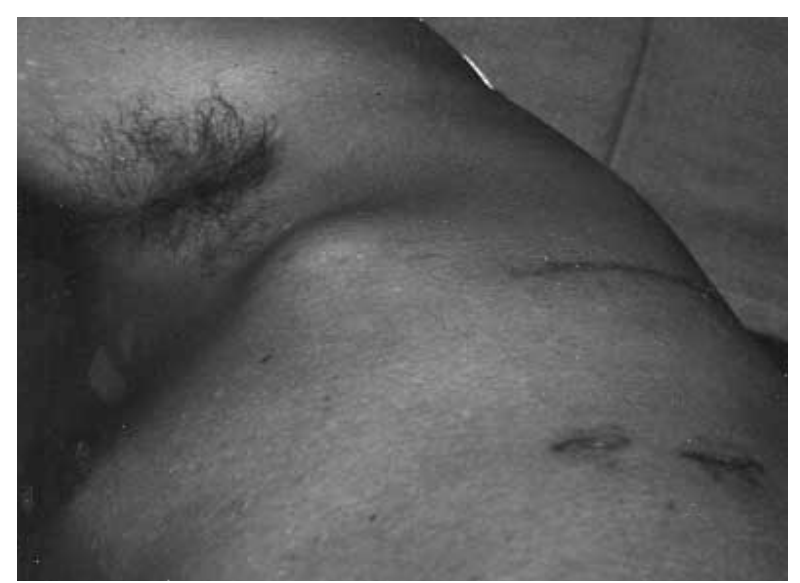

Fig 1. A protuberant mass is noted on physical examination in the anterior axillary line remote from the site of the previous thoracotomy and in the vicinity of the CT-guided needle biopsy.

At 6-month follow-up the patient reported a painful, expanding mass in the left chest wall. Physical examination showed only a $3 \times 3 \mathrm{~cm}$ firm, mobile mass over the anterior axillary line of the left chest wall approximately $3 \mathrm{~cm}$ superior to the previous thoracotomy incision (Fig 1). A chest radiograph showed normal lung parenchyma and no evidence of metastatic disease. Review of the preoperative CT-guided lung biopsy films showed an entrance tract in the immediate vicinity of this mass (Fig 2).

After bronchoscopy, the mass was excised and axillary nodes were sampled. Pathologic examination revealed a poorly differentiated $2.5-\mathrm{cm}$ adenocarcinoma with histologic findings similar to the more undifferentiated areas of the previous lung carcinoma. Specimen margins were microscopically free of malignancy.

A complete work-up showed no evidence of metastatic disease. The patient received adjuvant therapy including radiation (total of 5000 rads) and systemic carboplatin and paclitaxel. Six months after the operation, he has no evidence of metastatic or recurrent disease.

Comment. Needle aspiration biopsy is a well-established technique for cytologic diagnosis of malignant neoplasms. Tumor dissemination in the needle tract is a rare complication with an overall reported incidence of less than $1 \% .{ }^{3}$ Similar observations for cancer cell seeding have been reported with the use of VATS for the diagnosis and resection of primary lung carcinomas. ${ }^{4,5}$ As the risk of tumor implantation appears dependent on tract size, use of large-bore needles or thoracoscopy ports carries a relatively higher incidence of malignant seeding. 
The true incidence of tumor implantation along needle tracts in biopsy procedures might be higher than estimated, because many patients die before these metastases manifest themselves clinically. It has been suggested that patients with a solitary pulmonary nodule who are good operative candidates and amenable to a potentially curative resection would benefit from direct surgical exploration without preoperative percutaneous biopsy.

Treatment of soft tissue metastases after percutaneous needle biopsy involves surgical resection with chest wall reconstruction. ${ }^{3-5}$ Radical resection has not been proven superior to resection with negative surgical margins with regard to survival. Axillary lymph node dissection is deemed necessary when the tumor is in proximity to the axilla, because secondary metastases to axillary nodes have been reported. ${ }^{3}$ Postoperative radiation and chemotherapy are also given as adjuvant measures. We elected to treat this patient with complete but not radical (ie, 4-cm margins) surgical resection, local radiation, and systemic chemotherapy. The decision for local radiation and chemotherapy was based on the close margins of resection in an effort to control undetected micrometastases.

Percutaneous lung biopsy remains an effective alternative to exploratory surgery for tissue diagnosis of lung masses. Use of this diagnostic modality should be considered individually in light of operative risk as well as potential for curative resection.

\section{REFERENCES}

1. Libby DM, Henschke CI, Yankelevitz DF. The solitary pulmonary nodule: update 1995. Am J Med 1995;99:491-6.

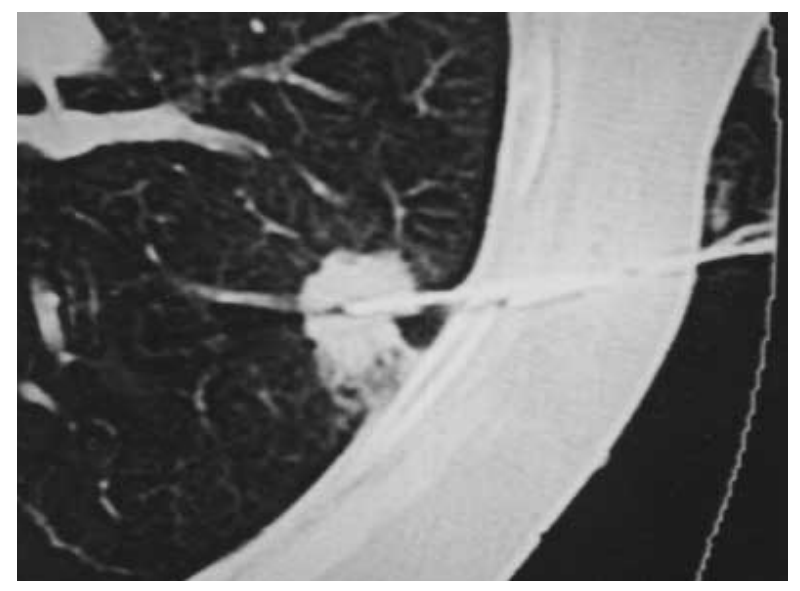

Fig 2. Initial preoperative CT-guided needle biopsy of the left upper lobe tumor.

2. Downey RJ, McCormack P, LoCicero J. Dissemination of malignant tumors after video-assisted thoracic surgery: a report of 21 cases. J Thorac Cardiovasc Surg 1996;111:954-60.

3. Seyfer AE, Walsh DS, Graeber GM, Nuno IN, Eliasson AH. Chest wall implantation of lung cancer after thin-needle aspiration biopsy. Ann Thorac Surg 1989;48:284-86.

4. Fry WA, Siddiqui A, Pensler JM, Mostafavi H. Thoracoscopic implantation of cancer with a fatal outcome. Ann Thorac Surg 1995;59:42-5.

5. Buhr J, Hurtgen M, Kelm C, Schwemmle K. Tumor dissemination after thoracoscopic resection for lung cancer. $\mathrm{J}$ Thorac Cardiovasc Surg 1995;110:855-6. 